\title{
SUMA DE NEGOCIOS
}

\section{Artículo de investigación}

\section{Símbolos, significados y prácticas asociados a las finanzas personales en estudiantes universitarios}

\author{
Samir Ricardo Neme-Chaves ${ }^{a}$ y Sara Catalina Forero-Molina ${ }^{b}$ \\ ${ }^{a}$ Magíster en Psicología del Consumidor, docente investigador Facultad de Mercadeo -Universidad Santo Tomás. Bogotá - Colombia. \\ (Autor de correspondencia) Correo electrónico: samirneme@usantotomas.edu.co. ORCID: 0000-0003-2327-4947 \\ ${ }^{b}$ Magíster en Administración, docente investigadora Facultad de Mercadeo - Universidad Santo Tomás. Bogotá - Colombia. \\ Correo electrónico: saraforero@usantotomas.edu.co. ORCID: 0000-0002-3020-0690
}

\section{INFORMACIÓN DEL ARTÍCULO}

Recibido el 26 de Abril de 2018

Aceptado el 7 de Junio de 2018

Online el 29 de Junio de 2018

\section{Códigos JEL:}

D14, D19, D31, I22

Palabras clave:

Símbolos, significados,

prácticas, finanzas

personales, universitarios.
Keywords:

Symbols, meanings,

practices,

personal finances,

university students.

\section{R E S U M E N}

El manejo adecuado del dinero es fundamental, y por ello las Finanzas Personales surgen como eje de apoyo. El presente estudio mediante la metodología cualitativa tiene como objetivo conocer los símbolos, significados y prácticas asociados a las finanzas personales por parte de estudiantes universitarios de Bogotá. Se utilizó un muestreo teórico con la participación de 25 estudiantes de universidades públicas y privadas, entre 18 y 24 años. Según los resultados, los jóvenes buscan experiencias de corto plazo que redundan en sus finanzas personales, sin despreocuparse por el ahorro o endeudamiento excesivo. Como conclusión, el enfoque educativo sobre finanzas personales requiere renovación en términos de las perspectivas, expectativas, gustos y necesidades de las nuevas generaciones.

Symbols, meanings and practices associated with personal finances in university students

A B S T R A C T

The adequate management of money is important, and therefore the Personal Finance emerges as axis of support. The present study through the qualitative methodology has as objective to know the symbols, meanings and practices associated to the personal finances on the part of university students of Bogota. A theoretical sampling was used with the participation of 25 students from public and private universities, between 18 and 24 years old. The results show that young people seek short-term experiences that result in their personal finances, without disregard for savings or excessive indebtedness. In conclusion, that the educational approach to personal finance requires renewal in terms of the perspectives, expectations, tastes and needs of the new generations. 


\section{Introducción}

Durante la vida, se plantean metas financieras que incluyen ahorrar, pagar deudas o equilibrar gastos e ingresos, sin embargo, hay falta de monitoreo sobre dichas acciones (Chang, Webb, Benn, y Reynolds, 2017), conllevando a la desarticulación entre objetivos y resultados. Así, en América Latina la población no ahorra para su jubilación, quienes tienen ingresos menores ahorran informalmente, y la inversión en acciones es más común en hombres con ingresos altos, en jóvenes y en los de mayor nivel educativo (Roa, Alonso, Bohórquez, y Rodríguez, 2014).

Ahora, las nuevas generaciones rompen esquemas tradicionales al buscar experiencias de corto plazo como viajes, adquisición de gadgets tecnológicos y artículos de moda, vivir con los padres, dilatar la decisión de casarse, de tener hijos y de comprar vivienda (Cutler, 2015; DeVaney, 2015; Gudmunson, Zuiker, Katras, y Sabri, 2015; Euromonitor, 2017; Klein y Smart, 2017), influyendo en sus finanzas personales.

Estas generaciones, Millennials (nacidos entre $1981 \mathrm{y}$ 2000) y Z (1993 - 2005), son más conscientes del ahorro (Turner, 2015), pero manifiestan alta desconfianza en el sistema financiero; en Colombia, diferentes medios de comunicación reiteran que los universitarios no saben manejar una tarjeta de crédito, creen que los productos bancarios son caros y tienen confusión en términos financieros; además, no están familiarizados con el ahorro y la inversión (Ministerio de Educación, 2011).

Así, aunque los jóvenes son la generación más educada en la actualidad, también son la más endeudada (Cutler, 2015; Cho, Xu y Kiss, 2015) y para la mayoría de los universitarios colombianos, que obtienen ingresos de sus padres, el gasto representa el 56\% en diversión, 34\% en necesidades básicas y académicas y 10\% en ahorro (Rebellón \& Salcedo, 2014).

El tema de los jóvenes y su economía ha sido bastante explorado. Según Denegri, Araneda, Ceppi, Olave, Olivares, y Sepúlveda (2016), los estudiantes reflejan menor impulsividad hacia la compra después de participar en un programa de manejo financiero. Otras investigaciones indican que el conocimiento financiero subjetivo influye más en la prevención de comportamientos riesgosos de crédito, que el financiero objetivo (Agnew y Harrison, 2015; Navickas, Gudaitis y Krajnakova, 2014).

Para Xiao, Tang, Serido y Shim (2011), Cho et al. (2015), Norvilitis y Mao (2013) y Hancock, Jorgensen y Swanson (2013), las normas paternas y la situación socioeconómica de los padres influyen en los comportamientos de riesgo crediticio de los estudiantes.

La postergación de la gratificación y la educación de los padres predicen la autoconfianza y el bienestar financiero; además el alto nivel de endeudamiento es predictor del abandono universitario, y las actitudes de mayor tolerancia al endeudamiento se relacionan con un locus externo en materia de consumo (Agnew y Harrison, 2015; Dwyer, Hodson y McCloud, 2013; Mansilla, Denegri y Álvarez, 2016). Así, Amar, Abello, Denegri, y Llanos (2007), evidencian que solo el $24 \%$ de los universitarios alcanzan el nivel de pensamiento económico inferencial, y no hay diferencias significativas de acuerdo con el programa académico al que pertenecen.
Particularmente, Neme-Chaves y Forero (2017) manifiestan que los países más productivos en el tema de finanzas personales en jóvenes son Estados Unidos, Reino Unido y Australia, y concluyen que falta profundidad en el estudio de esta temática.

En consonancia, los bajos niveles de alfabetismo financiero han sido preocupantes por las implicaciones que impactan más allá de lo individual (Atkinson y Messy, 2014). Así, la educación financiera es constante en las agendas de los países, buscando la reducción de la exclusión social, generación de bienestar y desarrollo del sistema financiero (Farrel, Fry, y Risse, 2016). Por ende, recientemente los bancos centrales y las superintendencias de los países latinoamericanos se han interesado más por las experiencias internacionales en inclusión y educación financiera (Roa et al., 2014).

En Colombia, actualmente hay 113 instituciones ejecutando 290 iniciativas de educación económica y financiera, principalmente en escuelas, colegios, y universidades (CIEFF, 2017). Se destacan la Bolsa de Valores de Colombia, el Ministerio de Educación Nacional, Banca de Oportunidades, Asobancaria, el Ministerio de Hacienda y Crédito Público, el Sena, la Superintendencia Financiera de Colombia, y los bancos de La República, Av Villas, Agrario de Colombia, de Bogotá y Citibank, entre otros, quienes desarrollan talleres, cursos, charlas, cartillas educativas y programas para niños, jóvenes y adultos (Ministerio de Educación, 2014).

Aún con los esfuerzos de cada país, persisten problemas en la estructura, evaluación e impacto del trabajo sobre alfabetismo y educación financiera. Para Castro y Fortunato (2015), no se ha estudiado profundamente la evolución del alfabetismo financiero en el ciclo de vida de los individuos frente al tiempo y cambios en el entorno, lo que impide tener mejores políticas y programas educativos.

De igual manera, según Raccanello y Herrera (2014) y Reniers, Beavan, Keogan, Furneaux, Mayhew \& Wood (2017), constantemente los programas de educación financiera descartan el análisis de patrones de comportamiento de tipo psicológico y emocional de los individuos en torno a sus finanzas personales, lo que complica el desarrollo de habilidades analíticas más enfocadas para tomar decisiones de largo plazo.

En concordancia, las finanzas tradicionales ven al inversionista como un individuo racional, sin embargo, las conductuales reconocen que existen aspectos psicológicos y emocionales que generan sesgos y riesgos en la toma de decisión hacia la inversión (Raccanello y Herrera, 2014).

Por lo mismo, el objetivo de la presente investigación es conocer los símbolos, significados y prácticas asociados a las finanzas personales por parte de universitarios de Bogotá, contribuyendo con la comprensión del conocimiento subjetivo de los estudiantes, y de las estrategias en el manejo de su dinero con miras a tener un soporte para programas de educación financiera mejorados. Este enfoque profundiza en aspectos más allá de la decisión racional de los jóvenes en el manejo de su dinero, reconociendo que, las finanzas personales no están separadas de la vida ética y moral (Brau, Nielson, y Sudweeks, 2015).

A continuación, se contextualiza lo referente a finanzas personales, símbolos y significados. El manejo del dinero es fundamental, y saber cómo guardarlo (ahorro), para qué 
sacarlo (gasto), cómo conseguir más empleando el que se tiene (inversión) y cuándo conseguir prestado (endeudamiento), son algunos de los componentes de las Finanzas Personales (Albornoz, 1998; Gudmunson et al., 2015; Chu, 2017).

Las finanzas personales son "el estudio y aplicación de herramientas de gestión financiera desde la perspectiva de una persona física" (Zicari, 2008, p.63); “comprenden la gestión de los ingresos, el presupuesto, el ahorro y el gasto de nuestro dinero en el tiempo, considerando los diferentes riesgos (...), con el fin de maximizar nuestro bienestar personal" (Chu, 2017, p.10).

Así, en Colombia se asocian los ingresos con las entradas de dinero o los recursos que pueden ser fijos (dinero que se recibe periódicamente y no varía significativamente en el corto plazo) o variables (no se reciben constantemente) (Melo, Téllez, \& Zárate, 2006).

El ahorro es la parte del dinero que no se gasta, sino que se guarda para necesidades futuras, imprevistos o como soporte a proyectos; puede ser informal (mediante alcancía u otro) o formal (mediante mecanismo regulado por una institución financiera) (Aibar, 2012).

En ocasiones, las personas recurren al endeudamiento para adquirir bienes y servicios, gastar o invertir (Aibar, 2012); a la suma de los créditos se le conoce como endeudamiento (Herranz \& Oro, 2017). En el sector financiero se define el crédito como el préstamo de dinero que se le otorga a una persona, entidad $u$ otro a alguien, con compromiso de devolverlo en el futuro junto con los intereses que represente; estos pueden ser informales (familia, amigos, etc.) o formales (por instituciones autorizadas y reguladas) (Asobancaria, Banca de las Oportunidades, \& Superintendencia Financiera de Colombia, 2017).

Particularmente, la inversión es la manera de poner a trabajar los ahorros generando rentabilidad y beneficios que se esperan recibir en el futuro (Atehortúa, 2012); los gastos son los usos que se le dan al dinero y se clasifican en los que satisfacen necesidades y en los que cumplen deseos; que pueden ser fijos (salidas de dinero permanentes) o variables (salidas de dinero difíciles de controlar).

Ahora, el símbolo es "un objeto que, aparte de su propia significación inmediata, sugiere también otra, especialmente de contenido más ideal, que no puede encarnar perfectamente" (Yepes y Aranguren, 2004, p. 251). Así, el símbolo se convierte en recurso para hacer presentes realidades que no puede o no quiere expresar claramente, lo que lo lleva a poseer lo simbolizado, por lo que el lenguaje simbólico permite la realización del ser humano como constructor de mundos (Vergel, 2014).

La función simbólica se da a partir de la imaginación y representa un papel fundamental para la cultura (Gutiérrez, Guerrero, Delgado, \& Vargas, 2017), reconociendo que los sistemas simbólicos van cambiando y variando con el contexto (Blumer, 2018; Eckert \& Nilsson, 2017; Sorensen, Tinc, Weil, \& Droullard, 2017).

Los símbolos se concretan a partir de acciones simbólicas que pretenden la utilización de un objeto o gesto simbólico acompañado por el recibimiento de un bien que es superior al símbolo, pero representado por él (Gutiérrez et al., 2017) y como indican Blumer (2018), Eckert \& Nilsson (2017) y
Sorensen et al. (2017), éstos pueden regir la conducta de los individuos expresando relaciones dominantes entre varios de ellos.

De acuerdo con Venkatesh (1999), en la comunicación los seres humanos usan signos y símbolos que otorgan significados. Así mismo, el marketing se caracteriza por la naturaleza simbólica de los procesos de consumo ya que "los símbolos crean significados y los consumidores negocian procesos a través de significados" (p. 5).

De igual modo, Ahmad, Al-Zboon, \& Dababneh (2018), Henry (2017), Key \& Noble (2017) y Post (2018), manifiestan que mediante signos y símbolos los individuos crean, transforman y mantienen su identidad propia a través del consumo de productos, de tal manera que el consumo es fuente de significados simbólicos con los cuales el ser humano sostiene su proyecto del yo (Tangsupwattana \& Liu, 2017). Asimismo, según Fenollar (2003), los autores que estudian el consumo más allá de la funcionalidad de los productos coinciden en que el consumidor hace uso de los símbolos que éstos poseen como forma de expresión social y/o personal.

Para Bruner (1998), el origen de los significados se da a nivel biológico y cultural ya que cuando éstos se entrelazan, el sujeto se apropia del lenguaje permitiéndole la construcción y entendimiento de los estos. Según Vygotsky (1995), es en el significado que el pensamiento y el habla se unen constituyendo un pensamiento verbal, razón por la cual "en el discurrir de las palabras es cuando se producen los significados" (Flórez, 2009, p.119).

\section{Metodología}

La metodología se soporta en el método del interaccionismo simbólico, entendido como una perspectiva sociológica teorizada por Blumer (1969) que subraya la centralidad analítica de investigar los significados que las personas dan a sus actividades, poniendo en relevancia la idea de que los individuos usan el lenguaje y los símbolos en su comunicación con los demás, construyendo la interpretación de puntos de vista subjetivos dando sentido a su mundo (Keller, 2005). Las premisas principales del enfoque son: (i) El ser humano orienta sus actos hacia las cosas en función del significado que tienen éstas para él; (ii) El significado de estas cosas u objetos surge como, consecuencia de la interacción social; (iii) Los significados se elaboran, reelaboran y modifican mediante un proceso interpretativo construido por la persona al enfrentarse con las cosas con las que se encuentra (Carter \& Fuller, 2015; Carter \& Fuller, 2016; Clarke y Keller, 2014).

Los participantes fueron seleccionados mediante muestreo teórico, hasta alcanzar saturación de los conceptos, participaron 25 estudiantes de universidades públicas y privadas, hombres y mujeres entre 18 y 24 años de edad, de diferentes semestres y carreras profesionales, que no estuvieran trabajando en el momento de las entrevistas, pero si lo estuviesen haciendo no fuera para sobrevivir.

Se realizaron 5 entrevistas y 4 sesiones de grupo con 5 estudiantes cada una, con una guía de 48 ítems, indagando sobre las categorías orientadoras del estudio: símbolos, significados y prácticas construidos respecto al ahorro, el 
gasto, la inversión y el endeudamiento. Por ende, la triangulación fue de métodos de recolección de información.

Inicialmente se diseñó el instrumento de recolección de información que fue evaluado por dos jueces expertos, cuyo concepto generó ajustes. Al inicio de cada sesión de grupo se administró el consentimiento informado a los participantes. Las sesiones de grupo y entrevistas procedieron con normalidad y el análisis de los datos se realizó con el software Atlas ti v.8.

\section{Resultado}

Respecto al ahorro (figura 1), los símbolos relacionados son la prevención de futuro, "El ahorro es como un colchoncito, en el momento que yo lo vaya a necesitar más adelante" (1:233). Además, simboliza cumplir sueños, metas, viajar, disfrutar, pasarla bien, pagar estudios: "pensar que en un futuro con ese dinero podré viajar, eso me motiva" (1:118).

La familia es la principal fuente de construcción de significados: "gracias a mi madre, es una persona que le gusta ahorrar, (...), desde muy pequeño me compraba alcancías con muñequitos" (1:246).
Por otro lado, la alcancía, mesa de noche, debajo del colchón o un libro, facilitan las prácticas de ahorro: "Mamá fue quien me enseñó a usar las alcancías y ahí empecé como un hábito" (1:204). Aunque los servicios bancarios facilitan la práctica, el significado asociado es que no existe un beneficio al usarlos: "de lo poco que averigüé la rentabilidad que te daban era muy baja, entonces pues, prefería tener mi dinero en el cuarto así no me estuviera dando rentabilidad" (1:250).

Así, ahorrar significa algo positivo para ellos y sus familias, pero expresan que es difícil y no significativo: "con la cantidad que uno tiene no sale bueno meter la plata en un banco" (1:159).

Respecto al gasto (figura 2), los símbolos son la satisfacción de necesidades y generación de felicidad momentánea: "En la vida universitaria hay mucho gasto y desperdicio de dinero" (1:7). También significa algo inevitable que la sociedad incita: "igualmente la sociedad digamos consumista también lo inclina a uno al gasto" (1:168).

Lo que impulsa las prácticas de gasto es el pago de los estudios universitarios, "bueno si tú pagas tus estudios, eso es bueno" (1:22), aunque también viajar, pasarla bien, y salir con amigos; ese tipo de gasto no se considera negativo

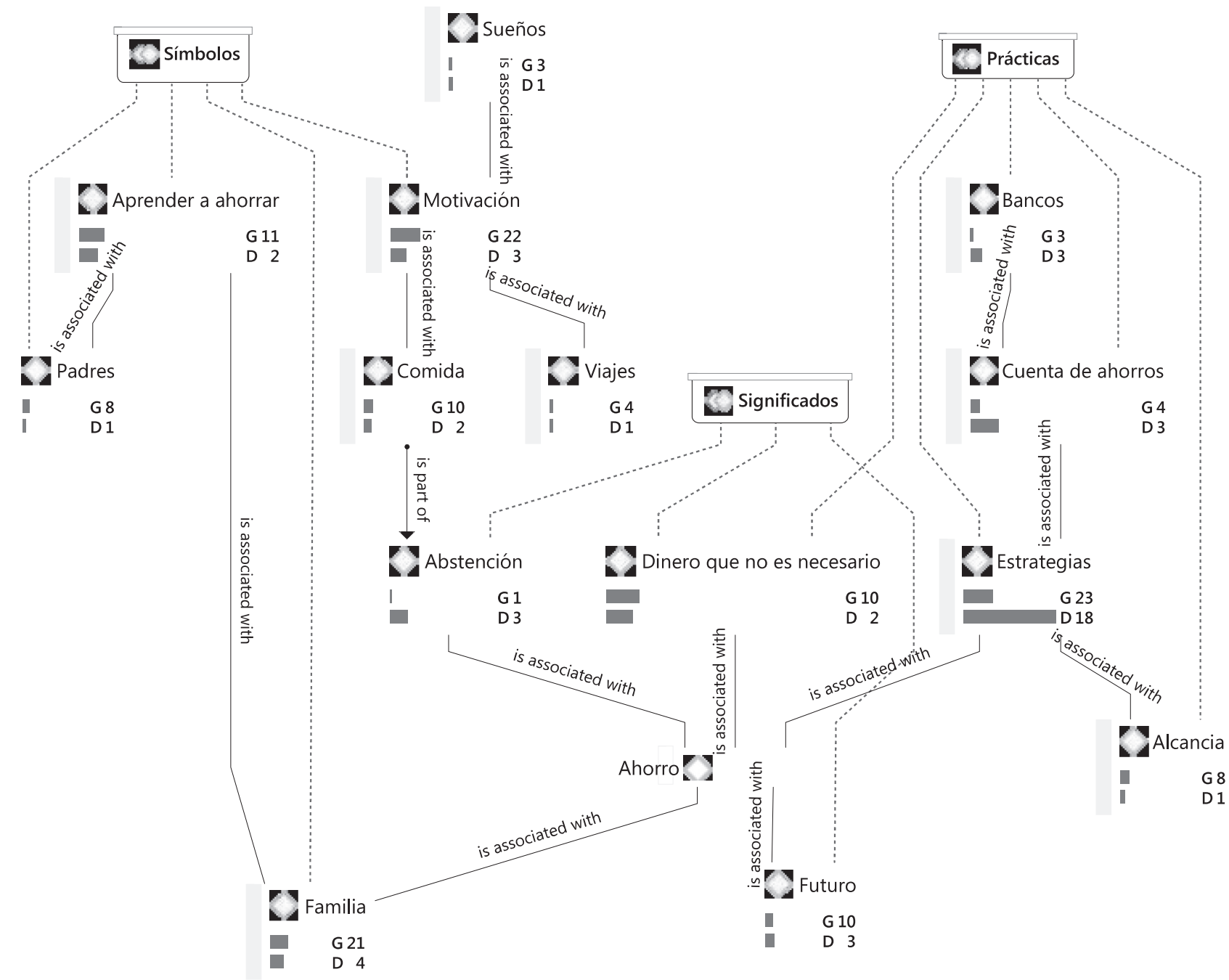

Figura 1 - Símbolos, significados, y prácticas respecto al ahorro 


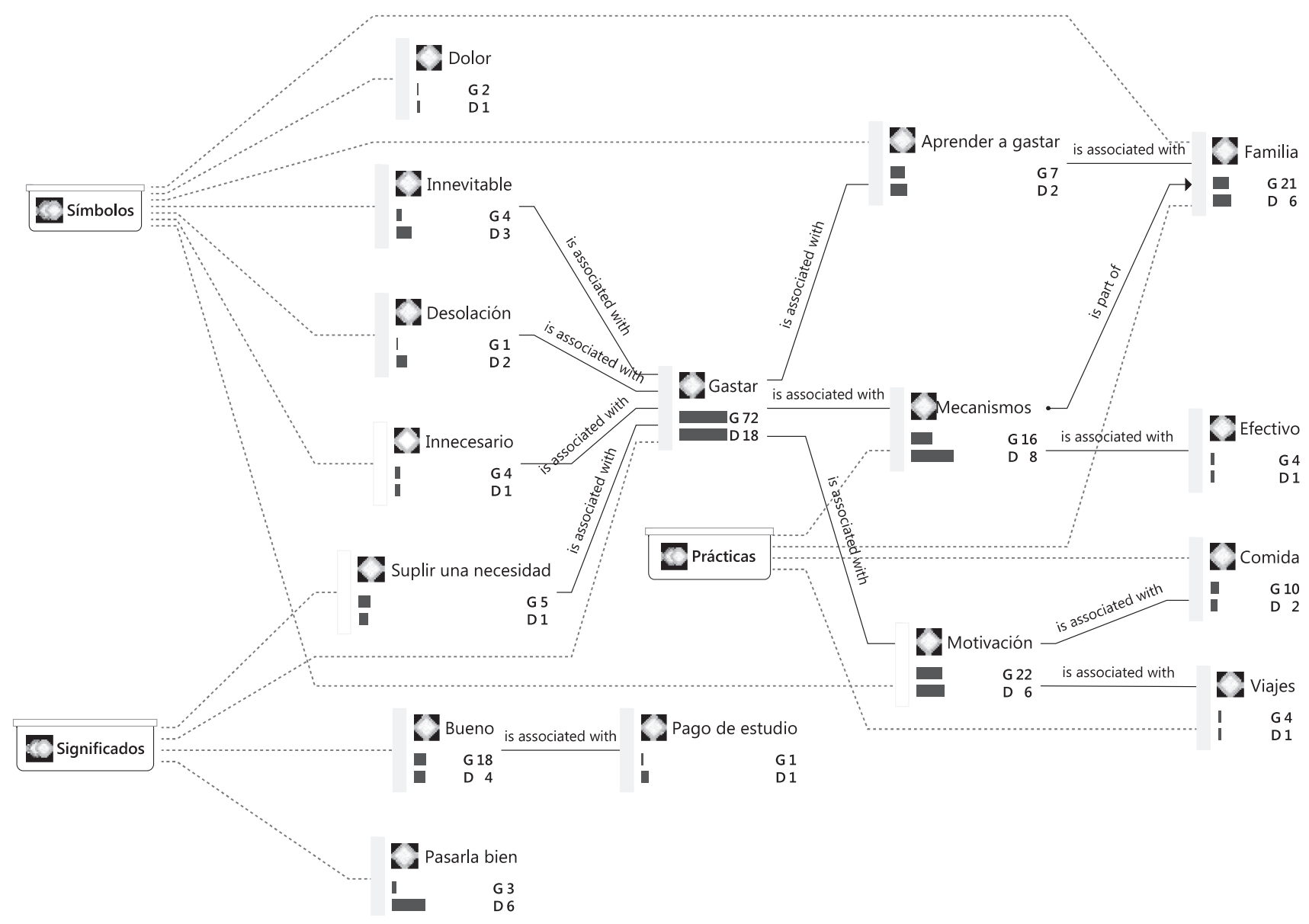

Figura 2 - Símbolos, significados, y prácticas respecto al gasto

Fuente: Elaboración propia

siempre y cuando no sea excesivo, "gastar en esencia en fiesta tampoco es malo el problema es gastar en exceso" (1:23).

El efectivo es el mecanismo más mencionado para la práctica de gasto: "Es mejor el dinero en efectivo, ahí lo que tienes” (1:112). Así, los símbolos relacionados con las tarjetas de crédito son temor y rechazo, por considerarlas una forma de gastar desmedidamente, "Yo estaría en la quiebra y reportada en datacrédito" (1:111).

La principal fuente de construcción de significados sobre el gasto es la familia: "En mi casa a uno le dicen si siente que si puede pagar uno decide si quiere o no" (1:191).

Los símbolos relacionados con el endeudamiento son negativos (figura 3): dolor y problemas; “(...) malestar en el caso que la persona no pueda pagarla y que (...) esté acosando que va a perder una casa, que va a perder sus bienes" (2:23). De otro lado, simboliza el cumplimiento de metas, invertir dinero, pagar la universidad, viajar, salir con amigos, pasarla bien, ir de vacaciones. También significa una oportunidad de tener dinero disponible rápidamente, y poder cubrir necesidades imprevistas: "Gastar algo que no tenía presupuestado" (1:186).

Los jóvenes conocen mecanismos para endeudarse como los bancos, los prestamistas o la familia, esta última es en la que más se apoyan porque no les cobran intereses: "es mejor ir con un tío que te preste los 3 millones de pesos que no te cobre interés a que uno ya irse con una entidad" (1:133); no corren ningún peligro, como si pidieran a un prestamista gota a gota: "un gota a gota, es muy riesgoso" (1:133).

La inversión para los estudiantes (figura 4) simboliza ingreso a largo plazo, ganancia en el futuro, un activo: "buscar otro ingreso a largo plazo, a futuro, tener una casa es invertir" (1:39).

Lo que impulsa la práctica de invertir es el no depender de un empleo, generar ingreso adicional, multiplicar el dinero, y seguridad financiera: "tener una seguridad financiera, un ingreso adicional, tener un ingreso constante" (1:49).

Las practicas se relacionan con negocios multinivel, compra de dólares, finca raíz, acciones y franquicias: "ahorita estamos mirando es en negocios multinivel" (1:46); "me gustaría o una inversión en finca raíz, (...) sí principalmente bolsa de valores" (1:221); "creo que en acciones, (...) a veces es un riesgo alto (...)" (1:274).

\section{Discusión y conclusiones}

Aquí se discuten los hallazgos en torno a los significados y símbolos que los participantes dan a sus prácticas dentro de las finanzas personales, interpretando desde el interaccionismo simbólico los puntos de vista subjetivos que le dan sentido a su actuar (Keller, 2005; Blumer, 1969). 


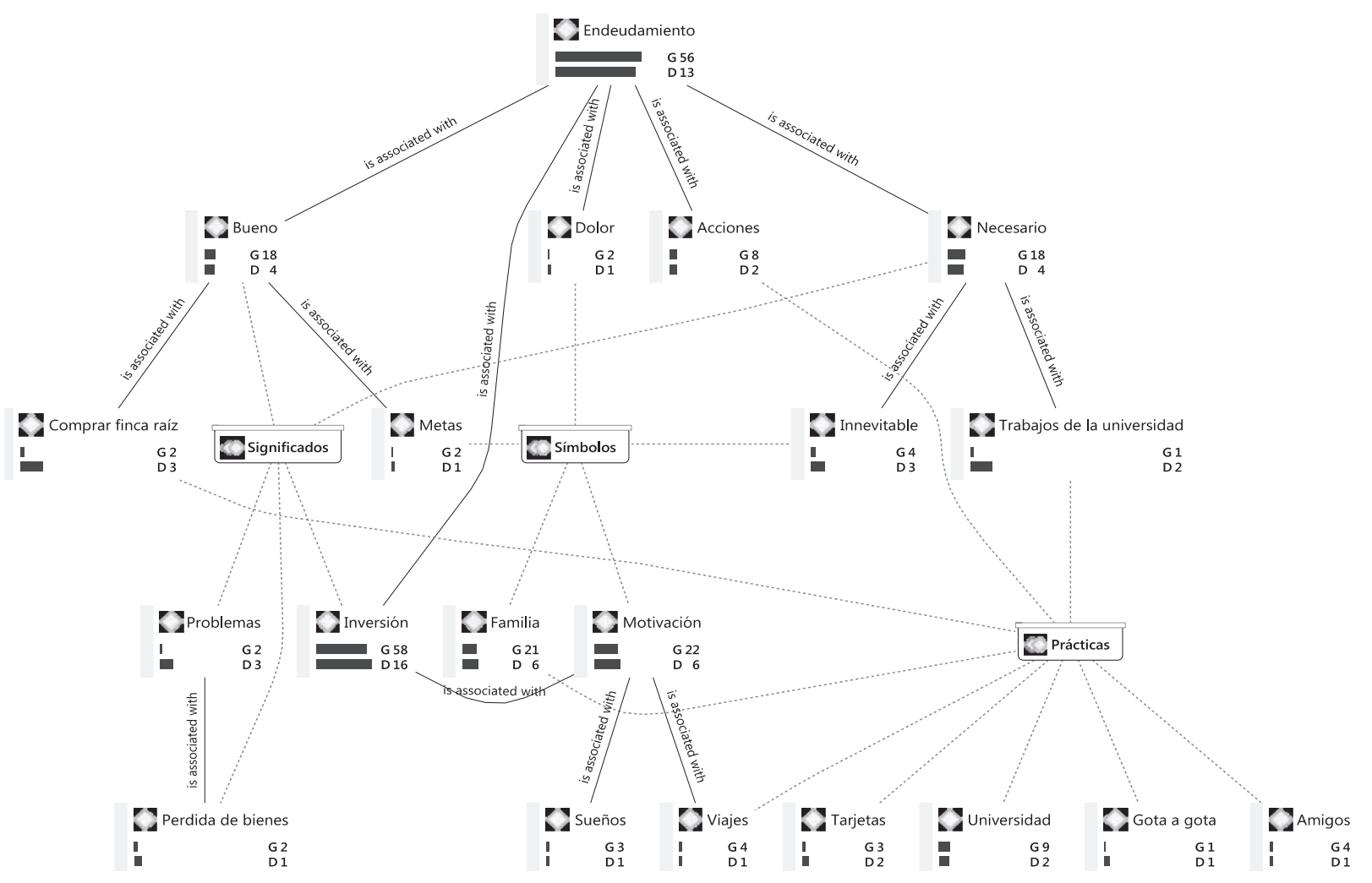

Figura 3 - Símbolos, significados, y prácticas respecto al endeudamiento

Fuente: Elaboración propia

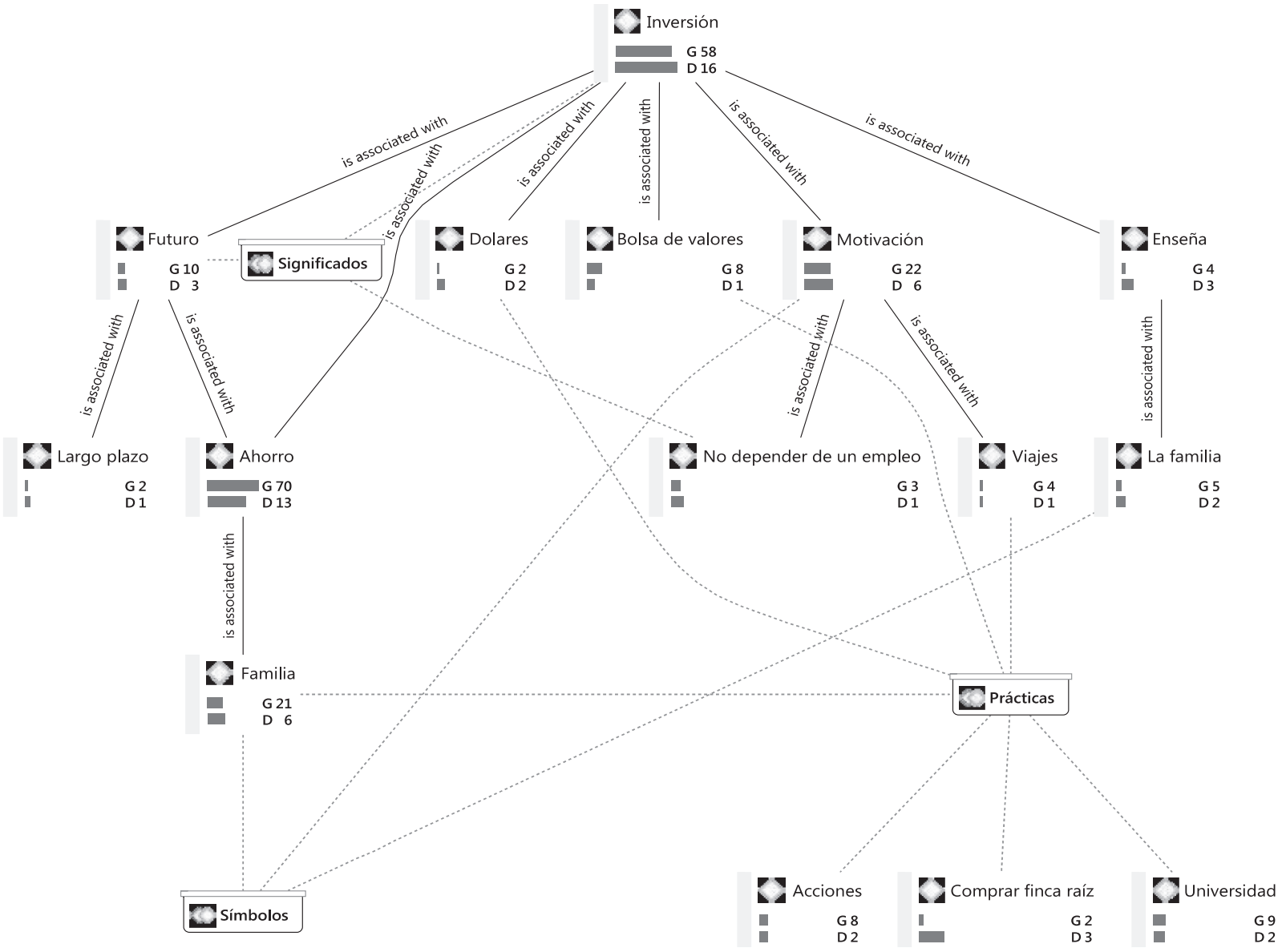

Figura 4 - Símbolos, significados, y prácticas respecto a la inversión

Fuente: Elaboración propia 
Los estudios sobre comportamiento de ahorro de los jóvenes exponen que ellos no son conscientes de la importancia de este (Ministerio de Educación, 2011), lo cual difiere con los resultados ya que sí lo consideran fundamental y coinciden con Aibar (2012), en cuanto a que el ahorro significa un soporte para necesidades futuras.

Para los participantes, el ahorro también simboliza el cumplimiento de sueños, metas y el pago de estudios; estos resultados se encuentran en línea con lo expuesto por Asobancaria et al. (2017).

De otro lado, los significados más sobresalientes entre los jóvenes respecto al ahorro son la consideración de algo positivo para toda la familia, ya que trae bienestar; sin embargo, es realmente difícil de lograr porque no cuentan con mucho dinero para ahorrar.

Para los estudiantes son mejores las prácticas de ahorro informales, coincidiendo con lo indicado por Roa et al. (2014) para el caso latinoamericano, ya que el significado hacia los bancos es que no traen beneficios por las deducciones hechas que son mayores a los réditos, y este comportamiento refleja la desconfianza por parte de los jóvenes expresada por Turner (2015). Así, ellos usan la alcancía y la mesa de noche para ahorrar. Se demuestra que la desconfianza en el sistema bancario es una de las razones por las que el significado asociado a éste es negativo.

Además, hay concordancias con lo postulado por Xiao et al. (2011), Cho et al. (2015), Norvilitis y Mao (2013), y Hancock et al. (2013) en cuanto a que las normas paternas influyen en los comportamientos financieros de los estudiantes, en otras palabras, para los participantes la familia es la principal fuente de construcción de significados sobre el ahorro, el endeudamiento y el gasto.

Por otro lado, para los universitarios el endeudamiento tiene símbolos negativos como pérdida de bienes, dolor y preocupación. Aunque ellos conocen mecanismos para la práctica como bancos y prestamistas, el más utilizado es la familia por el bajo riesgo que significa. Aseveran que para endeudarse hay que considerar el ingreso disponible y la capacidad de pago; esto permite interpretar que tal como lo manifiestan Agnew y Harrison (2015) y Navickas et al. 2014, el conocimiento financiero subjetivo influye más en la prevención de comportamientos riesgosos de crédito, que el financiero objetivo.

Del mismo modo, para los participantes endeudarse simboliza el cumplimiento de metas y la oportunidad de invertir el dinero (para el pago de estudios o cubrir imprevistos), sin embargo, también se asocia con símbolos como viajar, pasarla bien y divertirse, lo que está en conformidad con la tendencia presentada por Euromonitor (2017).

En cuanto al gasto, los estudiantes le asocian el símbolo de felicidad momentánea, lo que según Fenollar (2003) evidencia una manera de expresión social y/o personal, ya que para ellos significa algo inevitable porque la sociedad los incita a gastar, y a la vez evidencia la tensión y temor generada a nivel personal hacia el gasto sobre todo en mención de lo que las ocasionarían las tarjetas de crédito (endeudamiento excesivo).

Aun así, para ellos el gasto significa una forma de satisfacer necesidades, lo que lo convierte en un medio para realizar acciones de consumo cargadas de simbolismo, recibiendo bienes que serán superiores a los símbolos, pero representados por éstos (Gutiérrez et al. 2017). Sin embargo, los estudiantes no hacen una diferenciación entre los gastos para satisfacer necesidades y los relacionados con cumplir deseos (Asobancaria et al., 2017), ni los dividen entre fijos y variables.

Dichos bienes y/o servicios entran a responder a prácticas de gasto enfocadas en el pago de estudios universitarios, pero también en viajar, pasarla bien y salir con amigos, simbolizando algo positivo siempre y cuando no represente gastos excesivos. Esto se articula con la concepción de las Finanzas Personales para las cuales, el gasto implica saber para qué sacar dinero (Albornoz, 1998; Gudmunson et al., 2015; Chu, 2017), es decir, tener una justificación. Sin embargo, se puede evidenciar que a excepción de lo relacionado con los estudios, las demás son experiencias de corto plazo como lo dejan entrever Cutler (2015), DeVaney (2015), Gudmunson et al. (2015), Euromonitor (2017) y Klein y Smart (2017). De igual modo, se relaciona con lo mencionado por Rebellón \& Salcedo (2014), en cuanto a que la mayoría de los universitarios gastan más en diversión, seguido de necesidades básicas y académicas y en último lugar, en ahorro.

De la misma manera que Rebellón \& Salcedo (2014), en el presente estudio se evidencia que los estudiantes prefieren como mecanismo para la práctica del gasto el uso de dinero en efectivo y no las tarjetas de crédito, por tener hacia ellas temor y rechazo ante el símbolo de gasto desmedido que le asocian.

Finalmente, en lo referente a la inversión, los estudiantes le otorgan el símbolo de beneficio a largo plazo y ganancia en el futuro, incluso utilizan el término de activo; esto corresponde con la definición de inversión de Atehortua (2012).

En consonancia, los motivos que manifiestan los estudiantes para la práctica de invertir, si bien están articulados con la posibilidad de tener dinero para gastar en experiencias de corto plazo, también evidencian una preocupación por el futuro, utilizando términos como seguridad financiera; lo que a su vez, tiene sentido con los resultados que indican la actual conciencia sobre el ahorro por los estudiantes (Turner, 2015).

En cuanto a los mecanismos a través de los cuales se practica o se piensa practicar la inversión, los estudiantes coinciden con lo que Roa et al. (2014) manifiestan, ya que las acciones en Latinoamérica son una de las alternativas que están eligiendo los jóvenes y quienes tienen mayor nivel educativo. De igual manera, aunque según los resultados los jóvenes hablan de invertir, ya sea en acciones, en negocios multinivel, compra de dólares, finca raíz, franquicias y oro, ellos aseveran que existe riesgo; esto es consistente con el estudio de los autores citados por Raccanello y Herrera (2014), al afirmar que las finanzas conductuales reconocen que hay aspectos psicológicos y emocionales que generan riesgos en la toma de decisión hacia la inversión desde las finanzas personales.

Para finalizar, se evidencia que el objetivo planteado fue alcanzado con soporte en el método del interaccionismo simbólico, ya que se pudo dar razón de los símbolos y significados que los estudiantes universitarios participantes asocian con el manejo de sus finanzas personales y las prácticas derivadas, principalmente en términos del endeudamiento, el gasto, la inversión y el ahorro. 
De acuerdo con la primera premisa del método (Carter \& Fuller, 2015; Carter \& Fuller, 2016; Clarke y Keller, 2014), se puede concluir que los estudiantes universitarios orientan sus prácticas de finanzas personales soportándose en los significados que tienen para ellos el ahorro, gasto, inversión y endeudamiento, lo que de una u otra manera los impulsa a actuar, sin desconocer los temores y tensiones que se les presentan entre lo favorable y desfavorable de sus significaciones y lo fácil o difícil que puede ser la práctica.

En cuanto a la segunda premisa, se evidencia que para los estudiantes éstos significados se derivan de la interacción social ya que destacan sobre todo como fuente de aprendizaje la familia, principalmente para el endeudamiento, el gasto y el ahorro; y a la sociedad como incitador e impulsor de prácticas como el gasto. De igual manera, el hecho de asociar las prácticas con símbolos como el estudio, compartir con los amigos, viajar, cumplir sueños, entre otros, hace que se pueda deducir la conformación de éstos por el intercambio social ya que como menciona Flórez (2009), "en el discurrir de las palabras es cuando se producen los significados" (p.119).

Lo anterior resulta relevante teniendo en cuenta que se buscan experiencias de corto plazo en las nuevas generaciones, lo que se articula con lo postulado por Blumer (2018), Eckert \& Nilsson (2017), Sorensen (2017), en cuanto al reconocimiento de que los sistemas simbólicos van cambiando en conformidad con el contexto.

El tercer postulado se hace evidente en el análisis, ya que los estudiantes están constantemente elaborando y modificando los significados a través del proceso interpretativo que hacen al enfrentarse con las prácticas relacionadas con sus finanzas personales, brindando su percepción sobre cada uno de los ejes de indagación. Esto se articula, a su vez, con lo mencionado por Tangsupwattana \& Liu (2017), en tanto que toda la simbología en este tema es asociada por los estudiantes al consumo (viajes, experiencias, estudios, etc.), lo que deja entrever la interpretación soportada en significados simbólicos que se reflejan en su proyecto del yo.

Así, es evidente que el enfoque educativo sobre finanzas personales requiere una renovación en términos de las perspectivas, expectativas, gustos y necesidades de las nuevas generaciones, lo que conlleva el entendimiento de las dinámicas de una manera más equilibrada entre lo racional, psicológico y emocional.

A partir de este trabajo se sugieren futuras líneas de investigación que profundicen en estilos de vida y hábitos de consumo de las actuales generaciones, con el fin de aportar con profundidad a la responsabilidad a nivel individual y social que se tiene desde los programas de educación financiera y económica.

\section{Agradecimientos}

Queremos agradecer muy especialmente a las estudiantes Mayerli Medina, Michelle Escobar, Dayana Vargas y Juliana Moreno pertenecientes al semillero de Branding de la Facultad de Mercadeo de la Universidad Santo Tomás, por su gran ayuda a lo largo de este proyecto. La investigación presentada en este artículo fue financiada por la Universidad Santo Tomás de Colombia a través del Acta N 17130060 otorgada al primer autor.

\section{R E F E R E N C I A S}

Agnew, S. \& Harrison, N. (2015). Financial literacy and student attitudes to debt: A cross national study examining the influence of gender on personal finance concepts. Journal of Retailing and Consumer Services, 25, 122-129.

Ahmad, J., Al-Zboon, E. \& Dababneh, K. (2018). Children's recognition of pictorial signs and symbols. Early Child Development and Care, 188(6), 679-690.

Aibar, M. (2012). Finanzas personales: planificación, control y gestión. España: Ministerio de Educación de España.

Albornoz, G. (1998). Gestión Financiera Pública y el Municipio Colombiano. Cuadernos de Administración, 24, 183-193.

Amar, J., Abello, R., Denegri, M. \& Llanos, M. (2007). Pensamiento económico en jóvenes universitarios. Revista Latinoamericana de psicología, (39), 363-373

Asobancaria, Banca de las Oportunidades \& Superintendencia Financiera de Colombia (2017). Banca de las Oportunidades \& Superintendencia Financiera de Colombia. Educación financiera: construir, avanzar y prosperar. Recomendaciones para un buen manejo de las finanzas. (Babel, Ed.) Retrieved from https:// goo.gl/aL2SJj

Atehortúa, J.A. (2012). Mercado de capitales y portafolios de inversión. Medellín: Sello Editorial de la Universidad de Medellín.

Atkinson, A. \& Messy, F. (2014). Assessing Financial Literacy in 12 Countries. An OECD Pilot Exercise. Netspar. Retrieved from https://goo.gl/zaK9tp

Blumer, H. (1969). Symbolic Interactionism: Perspective and Method. USA: University of California Press.

Blumer, H. (2018). Industrialization as an agent of social change: A critical analysis. New York: Routledge.

Brau, J., Nielson, J. \& Sudweeks, B. (2015). Experiential Learning in Personal Finance: A Principles and Applications Based Approach. Journal of Financial Education, 41(2), 49-79.

Bruner, J. (1998). Actos de significado: más allá de la revolución cognitiva. Madrid: Alianza.

Carter, M. J. \& Fuller, C. (2016). Symbols, meaning, and action: The past, present, and future of symbolic interactionism. Current Sociology, 64(6), 931-961.

Carter, M. \& Fuller, C. (2015). Symbolic interactionism. Sociopedia. Isa, 1(1), 1-17.

Castro, R. \& Fortunato, A. (2015). ¿Se comporta el alfabetismo financiero como un bien económico?. Revista CEPAL, 116, 147162.

Chang, B., Webb, T., Benn, Y. \& Reynolds, J. (2017). Monitoring personal finances: Evidence that goal progress and regulatory focus influence when people check their balance. Journal of Economic Psychologic, 62, 33-49.

Cho, S. H., Xu, Y. \& Kiss, D. E. (2015) Understanding student loan decisions: A literature review. Family and Consumer Sciences Research Journal, 43(3), 229-243.

$\mathrm{Chu}$, M. (2017). Mis finanzas personales (Tercera ed.). Perú: Universidad Peruana de Ciencias Aplicadas.

CIEFF. (2017). Estrategia Nacional de Educación Económica y Financiera de Colombia (ENEEF).Retrieved from https://goo.gl/keAHDP

Clarke, A. \& Keller, R. (2014). Engaging complexities: Working against simplification as an agenda for qualitative research today. adele clarke in conversation with reiner keller. Forum: Qualitative Social Research, 15(2)

Cutler, N. (2015). Millennials and Finance: The "Amazon Generation". Journal of Financial Service Professionals, 69(6), 33-39.

Denegri, M., Araneda, K., Ceppi, P., Olave, N., Olivares, P. \& Sepúlveda, J. (2016). Alfabetización económica y actitudes hacia la compra en universitarios posterior a un programa de educación económica. REXE: Revista de estudios y experiencias en educación, 15(29), 65-81.

Devaney, S.A. (2015). Understanding the Millennial Generation. Financial Service, 69(6), 11-14 
Dwyer, R. E., Hodson, R. \& McCloud, L. (2013). Gender, debt, and dropping out of college. Gender \& Society, 27(1), 30-55.

Eckert, A. \& Nilsson, P. (2017). Introducing a symbolic interactionist approach on teaching mathematics: The case of revoicing as an interactional strategy in the teaching of probability. Journal of Mathematics Teacher Education, 20(1), 31-48.

Euromonitor, I. (2017) Millennials and Households: Opportunities and Challenges. Passport. Retrieved to: https://goo.gl/ moLHkY

Farrel, L., Fry, T. \& Risse, L. (2016). The significance of financial self-efficacy in explaining women's personal finance behaviour. Journal of Economic Psychology, 54, 85-99.

Fenollar, P. (2003). Estilos de vida: paradigma del mercado (Doctoral dissertation). Retrieved from https://www.tdx.cat/handle/10803/457807

Flórez, M. (2009). La construcción social de significados en el fin de la era del padre. Revista de Ciencias Sociales, 3(125), 117-125.

Gudmunson, C. G., Zuiker V. S., Katras, M. J. \& Sabri, M. F. (2015) Enhancing personal and family finance courses using case studies. College Student Journal, 49(3), 321-330.

Gutiérrez, S. I. N., Guerrero, R. F., Delgado, M. E. L. \& Vargas, M. G. O. (2017). Interaccionismo simbólico y teoría fundamentada: Un camino para enfermería para comprender los significados. Cultura De Los Cuidados: Revista De Enfermería Y Humanidades, (49), 190-199.

Hancock, A. M., Jorgensen, B. L. \& Swanson, M. S. (2013). College students and credit card use: The role of parents, work experience, financial knowledge, and credit card attitudes. Journal of Family and Economic Issues, 34(4), 369-381.

Henry, L. (2017). Language of the soul: When the soul speaks: The signs and symbols spirit uses to help us heal. Balboa Press.

Herranz, R. \& Oro, C. P. (2017). Las estructuras sociales de endeudamiento y los grupos de referencia comunitarios. Papers: Revista De Sociología, 102(1), 73.

Keller, R. (2005). Analysing discourse: An approach from the sociology of knowledge. Forum Qualitative Social Research, 6(3), 14.

Key, L. E. \& Noble, B. P. (2017). Course in general linguistics (1st ed.). London: Macat Library.

Klein, N. J. \& Smart, M. J. (2017). Millennials and car ownership: Less money, fewer cars. Transport Policy, 53, 20-29.

Mansilla, L., Denegri, M. \& Álvarez, B (2016). Relación entre actitudes hacia el endeudamiento y locus de control del consumidor en estudiantes universitarios. Suma psicológica, 23(1), 1-9

Melo, L., Téllez, J. \& Zárate, H. (2006). El ahorro de los hogares en Colombia. Revista Espe, (52), 110-161.

Ministerio de Educación. (2011). La influencia de la familia en los buenos hábitos financieros. Retrieved from: https://goo.gl/680 Jkg

Ministerio de Educación. (2014) Colombia formará a sus niños y jóvenes en educación económica y financiera. Retrieved from: https://goo.gl/TiS7VK
Navickas, M., Gudaitis, T. \& Krajnakova, E. (2014). Influence of financial literacy on management of personal finances in a young household. Verslas: teorija ir praktika, 1, 32-40

Neme-Chaves, S. R. \& Forero, S. (2017). Análisis bibliométrico acerca de las finanzas personales en estudiantes universitarios. In O. R. Group, Organizaciones Modernas para el Desarrollo Sostenible (pp. 22-38). Medellín: Optimal Research SAS.

Norvilitis, J. M. \& Mao, Y. (2013). Attitudes towards credit and finances among college students in China and the United States. International journal of psychology, 48(3), 389-398.

Post, W. E. (2018). Saints, signs, and symbols. Pickle Partners Publishing.

Raccanello, K. \& Herrera, E. (2014). Educación e inclusión financiera. Revista Latinoamericana de Estudios Educativos, XLIV(2), 119-141.

Rebellón, M. \& Salcedo, D. (2014). Origen, usos y significados del dinero en los universitarios bogotanos (Master's thesis). Available from intellectum unisabana

Reniers, R. L., Beavan, A., Keogan, L., Furneaux, A., Mayhew, S. \& Wood, S. J. (2017). Is it all in the reward? peers influence risk-taking behaviour in young adulthood. British Journal of Psychology, 108(2), 276-295.

Roa, M., Alonso, G., Bohórquez, N. \& Rodríguez, D. (2014). Educación e inclusión financieras en América Latina y el Caribe. Programas de los bancos centrales y las superintendencias financieras (Primera ed.). (Centro de Estudios Monetarios Latinoamericanos, \& Banco de la República de Colombia, Eds.) México.

Sorensen, J. A., Tinc, P. J., Weil, R. \& Droullard, D. (2017). Symbolic interactionism: A framework for understanding risk-taking behaviors in farm communities. Journal of Agromedicine, 22(1), 26-35.

Tangsupwattana, W. \& Liu, X. (2017). Symbolic consumption and Generation Y consumers: evidence from Thailand. Asia Pacific Journal of Marketing and Logistics, 29(5), 917-932.

Turner, A. (2015). Generation Z: Technology and Social Interest. The Journal of Individual Psychology, 71(2), 103 - 113.

Venkatesh, A. (1999). Postmodernism Perspectives for Macromarketing: An Inquiry into the Global Information and Sign Economy. Journal of Macromarketing, 19(12), 1-28.

Vergel, R. (2014). El signo en Vygotski y su vínculo con el desarrollo de los procesos psicológicos superiores. Folios, (39), 65-76.

Vygotsky, L. (1995). Pensamiento y lenguaje. Teoría del desarrollo cultural de las funciones psíquicas. Ediciones Fausto. Rusia.

Xiao, J. J., Tang, C., Serido, J. \& Shim, S. (2011). Antecedents and consequences of risky credit behavior among college students: Application and extension of the theory of planned behavior. Journal of Public Policy \& Marketing, 30(2), 239-245.

Yepes, R. \& Aranguren, J. (2004). Fundamentos de antropología: un ideal de la excelencia humana (Sexta ed.). Navarra: Eunsa.

Zicari, A. (2008). Finanzas personales y ciclo de vida: un desafío actual. Universidad del Centro Educativo Latinoamericano, 11(20), 63-71. 